\section{Revista dePolítica Económica y Desarrollo Sostenible}

Centro Internacional de Política Económica para el Desarrollo Sostenible
Revista de Política Económica y Desarrollo Sostenible

EISSN: 2215-4167 • Vol. 4 (2) • Enero-Junio, 2019: 1-19

DOI: http://dx.doi.org/10.15359/peds.4-2.1

URL: http://www.revistas.una.ac.cr/politicaeconomica

Revista electrónica semestral publicada por el Centro Internacional de Política Económica para el Desarrollo Sostenible

Universidad Nacional, Lagunilla, Heredia Apartado 2393-3000

\title{
Mercado virtual: ¿Cómo hacerlo más accesible y funcional para las PYMES?
}

Virtual Market: How to Make it More Accessible and Functional for SME'S?

Raúl Fonseca Hernández ${ }^{1}$

(iD http://orcid.org/0000-0002-4133-9591

Jonathan Porras Quesada

Luana Vargas Chavarría

Paola Villalobos Solano ${ }^{4}$

Dirección de contacto:

raul.fonseca.hernandez@una.cr

Fecha de recibo: 26 de setiembre del 2018. Fecha de corrección: 28 de diciembre del 2018.

Fecha de aceptación: 14 de febrero del 2019. Fecha de publicación: 28 de febrero del 2019

\section{Resumen}

El artículo pretende identificar factores competitivos y elementos funcionales que deberían incorporar o mejorar las plataformas de mercados virtuales para hacerlas más accesibles y adaptadas a las necesidades de las pymes. Con tal propósito, se aplicó una encuesta a empresarios que habían utilizado alguna plataforma de mercado virtual y se analizaron ocho sitios nacionales y, principalmente, internacionales dedicados a tal fin. Se determina que las instituciones a cargo de los mercados virtuales deben diseñar una estrategia a corto y mediano plazo para su mejoramiento continuo, que incluya alguno de los siguientes elementos: renovar el diseño y ofrecer el contenido en varios idiomas; mejorar el registro y perfil de las empresas; ofrecer el servicio gratuito y financiar los gastos operativos por medio de publicidad o incluir funciones tendientes a mejorar la búsqueda, la negociación y el concretar negocios. El artículo concluye que los mercados virtuales son una opción viable para mejorar la competitividad de las empresas; sin embargo, los mercados virtuales que se creen o los existentes que están a disposición de las pymes de Costa Rica, deben ser más accesibles en términos funcionales y de costos de suscripción, así como contener funciones adaptadas a las necesidades de estas.

Palabras claves: marketing; comercio electrónico; Internet; competencia económica.

1 Académico e investigador, Escuela de Relaciones Internacionales, Universidad Nacional, Costa Rica y de la Sede de Occidente de la Universidad de Costa Rica. Máster en Economía del Desarrollo.

2 Procurement to Payment Specialist en Procter \& Gamble. Licenciado Relaciones Internacionales con énfasis en Comercio Internacional; jonpoque@hotmail.com

3 Asistente Administrativa - Contable en Embajada de Chile en Costa Rica. Licenciada Relaciones Internacionales con énfasis en Comercio Internacional; luanavargas20@gmail.com

4 Asistente Administrativa, Gerencia Franquicias Musmanni (Florida Retail). Licenciada Relaciones Internacionales con énfasis en Comercio Internacional; pao_tati06@hotmail.com 


\begin{abstract}
This article identify the competitive factors and functional elements that virtual markets should incorporate to make it more accessible and adapted to SME's needs. A survey was applied to entrepreneurs who had used virtual market platforms; eight national and international virtual market sites were analyzed. The results shows that institutions which hold virtual markets must to design a short and a medium-term strategy for their continuous improvement. Those strategies has to include: renewing design, contents in different languages, improving the company's profile and registration process, offering free virtual platform services, financing operational expenses through publicity, and improve searching, negotiating and business close tools. The research concludes that virtual markets are a viable option to improve the competitiveness of SMEs, however, in Costa Rica those markets must to be more accessible, in order to include functions adapted to SME needs.
\end{abstract}

Key words: marketing, online commerce, Internet, Economic competition

\title{
Introducción
}

Las pequeñas y medianas empresas (pymes) en Costa Rica tienen un peso importante en la economía del país. Según censos del Ministerio de Economía, Industria y Comercio (MEIC), para el año 2016, el 85,7 \% del parque empresarial en el país eran pymes. Estas, por su parte, generaron el $27 \%$ del empleo total del sector privado y representaron en 2016 el $16 \%$ del total de las exportaciones FOB (MEIC, 2017).

Las pymes se enfrentan a limitaciones tanto internas como externas que dificultan su desarrollo y consolidación en el mediano y largo plazo. Según el Estado de la Nación (2018) y un estudio realizado por el Observatorio del Desarrollo de la Universidad de Costa Rica (UCR, 2018), a nivel interno poseen deficiencias en conocimientos técnicos y empresariales, acceso a financiamiento, ineficiencias productivas, así como niveles de formalización parciales, pues son “semiformales", acuñando el término del Estado de la Nación (2018, p. 107), entre otros aspectos.

A nivel externo, la política pública y el contexto no siempre ofrecen el apoyo que requieren las empresas. El país implementó la política pública “Costa Rica Emprende” en 2010-2014; sin embargo, según Fonseca y Chaves (2015), su impacto no ha sido el esperado. Por ejemplo, la política no ha logrado estabilizar en el tiempo las pymes exportadoras (Sáurez y Fonseca, 2014) ni ha permitido aumentar el valor agregado (Chaves y Fonseca, 2015) que generan; además, presentan varios factores que dificultan su internacionalización (Tabares, Anzo y Vanegas, 2016), tales como "baja producción, poco conocimiento de los procesos de exportación, falta de información sobre mercados internacionales, insuficientes capitales de trabajo, ausencia de tecnología, deficiente control de calidad" (Varado, Cortez y Sánchez, 2018, p. 997).

Asimismo, el sistema nacional de innovación posee un marco normativo robusto, pero debe avanzar en aumentar la cobertura, acceso e impacto. En cuanto al Sistema de Banca para el Desarrollo, este posee una normativa fuerte, pero tiene una concentración de recursos, con baja utilización (Bonilla y Mesén, 2013) y con requisitos poco accesibles al empresario promedio (Fonseca y Chaves, 2015). 
En Costa Rica existen las condiciones básicas para la utilización de mercados virtuales para la venta y compra de bienes y servicios, siendo esta una forma viable de aumentar la competitividad de las empresas. Según un estudio de América Economía Intelligence, el comercio electrónico en América Latina y el Caribe creció un 42,8 \% durante el 2010 y 2011, debido a factores como mayor seguridad y confianza, plataformas comerciales generadas a partir de redes sociales, mejoras en las conexiones a Internet, aumento del uso de medios de pagos electrónicos, entre otros (Procomer, 2015). En este sentido, se evidencia que en los últimos cinco años existe un aumento importante en la utilización por parte de las pymes de computadoras e Internet; según el MEIC. en el 2013, el 68 \% contaba con un equipo de cómputo, mientras que un 66 \% tenía acceso a Internet. Transcurrido un lustro, en 2018, la tenencia de equipo de computación aumentó a 85 \% y la cantidad de empresas con acceso a Internet aumentó a un $82 \%$, con un promedio de velocidad de 10 GB (UCR, 2018). Estas condiciones proporcionaron mayor viabilidad al desarrollo y fortalecimiento de los mercados virtuales en el país.

Las investigaciones actuales se centran mayoritariamente en el desarrollo del comercio electrónico a nivel general, pero no necesariamente en el estudio de un mercado virtual o marketplace de forma específica. Es decir, el mercado virtual es una forma o una herramienta del comercio electrónico como categoría de análisis integradora. Autores como Nieto (2016), Miño, Napoleón y Orozco (2017) y Hernández (2017), resaltan la importancia del comercio electrónico y la creación de planes de marketing digital, la implementación de páginas web, blogs, redes sociales, sitios para realizar compras o encargos en la página de la empresa, o bien, el pago de publicidad en plataformas como Google, Facebook, correos masivos, entre otras estrategias. En esta línea, Espinoza y Flores (2018) definen el comercio electrónico como la estrategia o herramientas “encargadas de la compraventa de productos y servicios de manera electrónica" (p. 3).

Se evidencia un área de estudio interesante al analizar un mercado virtual como herramienta de comercio electrónico, así como, contar con una visión actual sobre elementos de política pública que podrían aplicarse en una estrategia de apoyo a las empresas que buscan su internacionalización. Para el caso de Costa Rica, existe un limitado análisis de casos de mercados virtuales como tales (lugares que confluyen oferentes y demandantes, pudiendo realizar negociaciones y compras en línea) (Lavios, Olmo, Mariscal, y García, 2005; Hurtado, 2009). De tal forma que se evidencia la necesidad de contar con un documento que identifique los factores competitivos y funcionales de las plataformas de mercados virtuales que puedan ser impulsados por acciones de política pública para la mejora de la competitividad de las pymes en Costa Rica.

La investigación pretende responder las siguientes interrogantes: ¿cuáles son las principales plataformas electrónicas que ofrece el servicio de mercado virtual al que empresas nacionales pueden acceder? y ¿cuáles factores competitivos y elementos funcionales deberían incorporar o mejorar las plataformas de mercados virtuales para hacerlos más accesibles y adaptados a las necesidades de las pymes?

El artículo pretende identificar factores competitivos que deberían incorporar o mejorar las plataformas de mercados virtuales para hacerlos más accesibles y adaptados a las necesidades de las pymes. 


\section{Referente teórico}

Para mejorar la competitividad de las empresas, la estrategia debe considerar tanto elementos vinculados con los insumos productivos, como otros aspectos intangibles. Autores como Varian (1998) indican que una empresa es competitiva cuando logra disminuir costos y maximizar ganancias, haciendo una clara alusión a que la empresa puede ser competitiva si mejora la utilización de los medios de producción. Una visión diferente tienen los autores Romo y Andel (2005), quienes indican que la competitividad se logra al acompañar el mejor uso de los insumos con la inclusión de "la noción de otros activos tangibles e intangibles en forma de tecnología y habilidades administrativas que, en su conjunto, actúan para incrementar la eficiencia en el uso de los insumos" (p. 201). Es precisamente esta extensión del concepto la que se adapta a los nuevos modelos productivos y de desarrollo de las empresas en los que se involucra no solo el insumo tradicional, sino que también se apoya la producción con las tecnologías y la gama de facilidades que estas ponen a disposición de las pymes en tiempos actuales.

En este sentido, la participación de las PYMES en mercados virtuales es una estrategia que apoya la mejora de la competitividad, en el sentido propuesto por Romo y Andel (2005). Como punto de partida, Lavios, Olmo, Mariscal y García (2005) definen la herramienta tecnológica del mercado virtual como "un sitio web en el que se ofrecen funciones comerciales a empresas que acuden a él para comprar o vender sus productos. El mercado electrónico asume ciertas funciones comerciales, como la negociación de precios o la presentación de catálogos” (p. 1). Los autores presentan una definición que abarca acciones más diversas y que comprende todas aquellas interacciones gestionadas mediante un sitio web o herramienta, con el fin de establecer vínculos comerciales y mejoras consecuentes en la competitividad de las pymes.

Ahora bien, un mercado virtual tiene alcances y limitaciones, Petronio (2003) ofrece las siguientes características:

... un mercado virtual, así como un mercado real, es un lugar donde si bien están definidas las reglas del juego, no lo están a priori, de qué forma o en qué condiciones se van a cerrar las transacciones. De hecho, las grandes transacciones requieren negociaciones complejas y los resultados distan mucho de la forma "vendo este producto a este precio". (p. 23)

De tal forma que la utilización de un mercado virtual requiere una madurez y competitividad de la pyme particular. Como indica el autor, las ventas por medio de plataformas virtuales requieren no solo un buen producto, sino también una experiencia adecuada por parte de la empresa productora, altos estándares de calidad, así como competencias en la utilización de tecnologías de la información y habilidades blandas como la negociación (Medina-Chicaiza, Tibanta-Narváez y Pazmay-Pazmay, 2017; Salas, Martínez y Chamba, 2018). En este sentido, Castro (2014) y Sarcos e Indriago (2016) advierten que en los mercados virtuales, al ampliarse la positividad de interacción entre empresas de diversos países, se complican las negociaciones 
en este tipo de ambientes, a causa de factores culturales, barreras idiomáticas y aspectos de normativa de acceso a mercados que deben solventarse para completar la transacción.

Para mejorar el aprovechamiento potencial de los mercados virtuales por parte de las pymes, se requiere una política de apoyo estatal. Al respecto, autores como Ruano (2016) y Figal y Maffioli (2016) argumentan que el Estado podría facilitar la plataforma y las capacitaciones y diseñar sistemas de incentivos, tanto monetarios como de beneficios intangibles, para los demandantes y los oferentes que se inscriban y transen bienes y servicios por medio de la plataforma. Además, se podrían generar programas subsidiados de acceso a paquetes básicos de Internet o compra de dispositivos móviles. Sin estos elementos, la incursión en los mercados virtuales serán aspectos esporádicos e, incluso, únicos.

\section{Metodología}

La identificación de las variables y los elementos a considerar en la mejora de las plataformas virtuales se realizó mediante los siguientes dos métodos: una encuesta aplicada a empresarios que han utilizado un mercado virtual para vender o comprar bienes y servicios en los últimos cinco años; y un análisis comparativo de ocho plataformas que ofrecen el servicio de mercado virtual, tanto a nivel nacional como internacional.

La base de datos para la realización de la encuesta fue suministrada por la Promotora de Comercio Exterior (Procomer), con datos al 2016, compuesta por 917 registros. Se entrevistó a una muestra de 47 empresarios (estadísticamente significativa al $85 \%$ de confianza). Los sujetos de estudio se seleccionan por medio de la técnica aleatoria, con remplazos elegidos bajo el mismo método, en caso de no obtener respuesta al tercer envío del correo electrónico y segunda llamada telefónica realizada. La encuesta se realizó con el apoyo de la herramienta Google Formularios. La información se levantó entre los meses de noviembre 2016 y enero 2017.

Como la principal limitación de la investigación, se debe apuntar que la base de datos suministrada tenía problemas con la actualización de los datos de contacto de las personas empresarias; no obstante, esta era la forma más conveniente de obtener información sobre pymes exportadoras que utilizaran plataformas de mercados digitales.

Las variables consideradas para la investigación toman como referencia los documentos de Reyes y Zamora (2009) y Hurtado (2009). En relación al primero, se afirma que el diseño, el proceso de desarrollo del sistema y el uso de sistemas web son los factores que se deben medir para determinar la efectividad de un sitio electrónico creado para promover el comercio. Así pues, se consideran como indicadores: los "parámetros de efectividad, productividad, seguridad y satisfacción de usuario" (González y Olsina citado por Reyes y Zamora, 2009, p. 7). Por su parte, el documento de Hurtado (2009) presenta como factores de análisis: la facilidad de aprendizaje, la eficiencia, la retención sobre el tiempo, tasas de error, así como la satisfacción subjetiva. 
El instrumento aplicado contó mayoritariamente con preguntas cerradas respecto a la utilidad de las herramientas de mercados virtuales, funcionalidades, resultados obtenidos al utilizar la plataforma, seguimiento que brindan los sitios utilizados, servicios complementarios ofrecidos y disposición a pagar por utilizar el servicio. Asimismo, se integran preguntas abiertas sobre las fortalezas, debilidades y oportunidades de mejora de las plataformas que se utilizan.

En cuanto al análisis comparativo, se analizaron diversas plataformas y diversos directorios empresariales a nivel nacional y, principalmente, internacional. El estudio se centró en sitios web identificados mediante una búsqueda secundaria y a partir de lo mencionado en la encuesta aplicada a los empresarios. Se seleccionan ocho que se consideran accesibles a las pymes nacionales y que permitieran la compra y venta de bienes y servicios. Se analizaron variables como diseño, idioma, costo, país, propiedad (pública o privada), sectores a los que se enfoca, opción para realizar compras en línea, publicidad, así como la dirección del sitio web. Estas variables se determinaron mediante una consulta a un grupo de expertos y el análisis de las plataformas en estudio, estableciendo elementos en común o diferenciadores entre estas.

Las recomendaciones emitidas para mejorar una herramienta de mercado virtual giran en torno a la información recabada por los métodos mencionados anteriormente y a su validación correspondiente con un grupo de expertos de la Universidad Nacional y de Procomer, realizada a finales de 2017.

\section{Análisis de los resultados}

La presente sección se divide en dos apartados. En el primero se muestran los principales resultados de la encuesta aplicada a empresarios que han utilizado alguna plataforma de mercado virtual y, en el segundo, el análisis de las diferentes plataformas de mercados virtuales. La tercera sección presenta los elementos que debería considerar una plataforma de mercado virtual para mejorar la competitividad de las empresas.

\section{a) Resultados de la encuesta aplicada}

La encuesta se aplicó a un total de 47 empresarios que han utilizado alguna herramienta de mercado virtual. La actividad económica de las empresas es diversa, principalmente se dedican a actividades de procesos, infraestructura, producción de materia prima. En menor medida, se ubican las empresas que compran y venden servicios de capital humano, administración y logística. El gráfico 1 muestra los porcentajes según actividad económica reportada en la encuesta. 
Revista de Política Económica y Desarrollo Sostenible • EISSN: 2215-4167

Vol. 4 (2) • Enero-Junio, 2019: 1-19

Fonseca Hernández

Porras Quesada

DOI: http://dx.doi.org/10.15359/peds.4-2.1

Vargas Chavarría

URL: http://www.revistas.una.ac.cr/politicaeconomica

Villalobos Solano

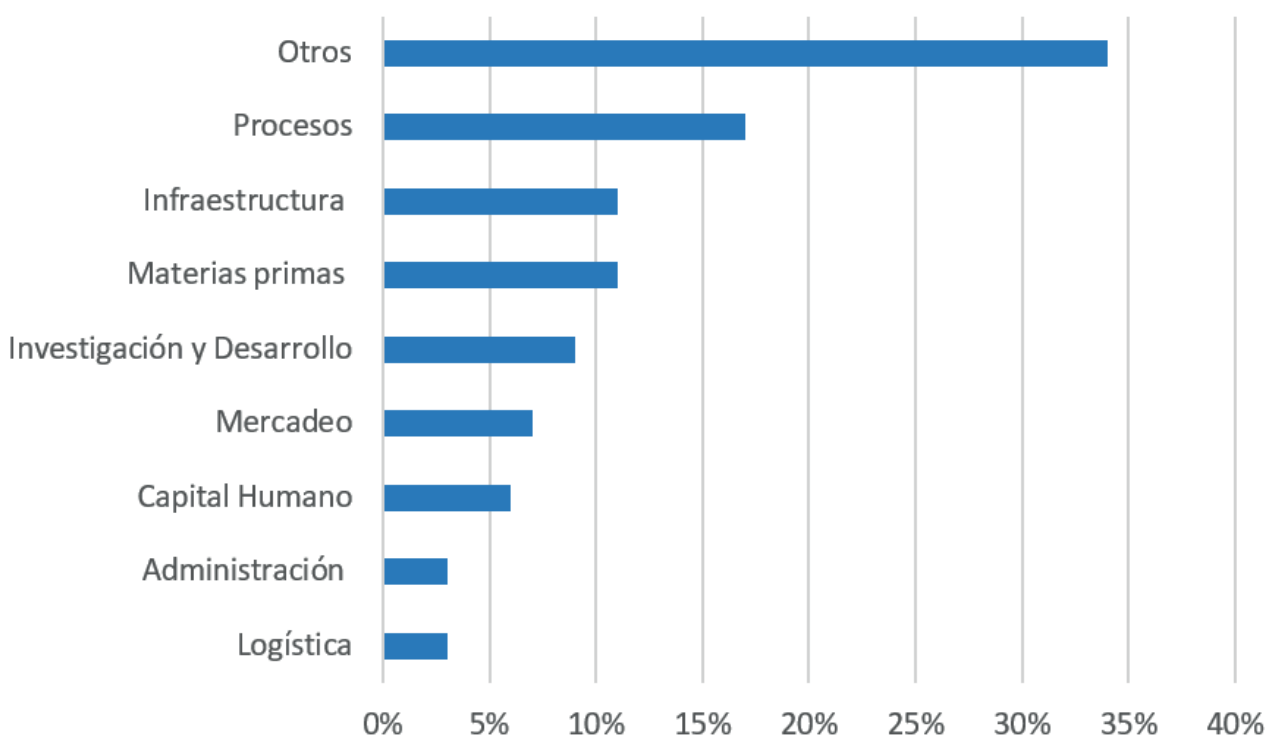

Gráfico 1. Actividad económica a la que se dedican las empresas entrevistadas Fuente: elaboración propia.

Los entrevistados pertenecen principalmente a la Gran Área Metropolitana. Menos del $5 \%$ de las empresas son extranjeras. El gráfico 2 muestra la distribución por ubicación de la empresa.

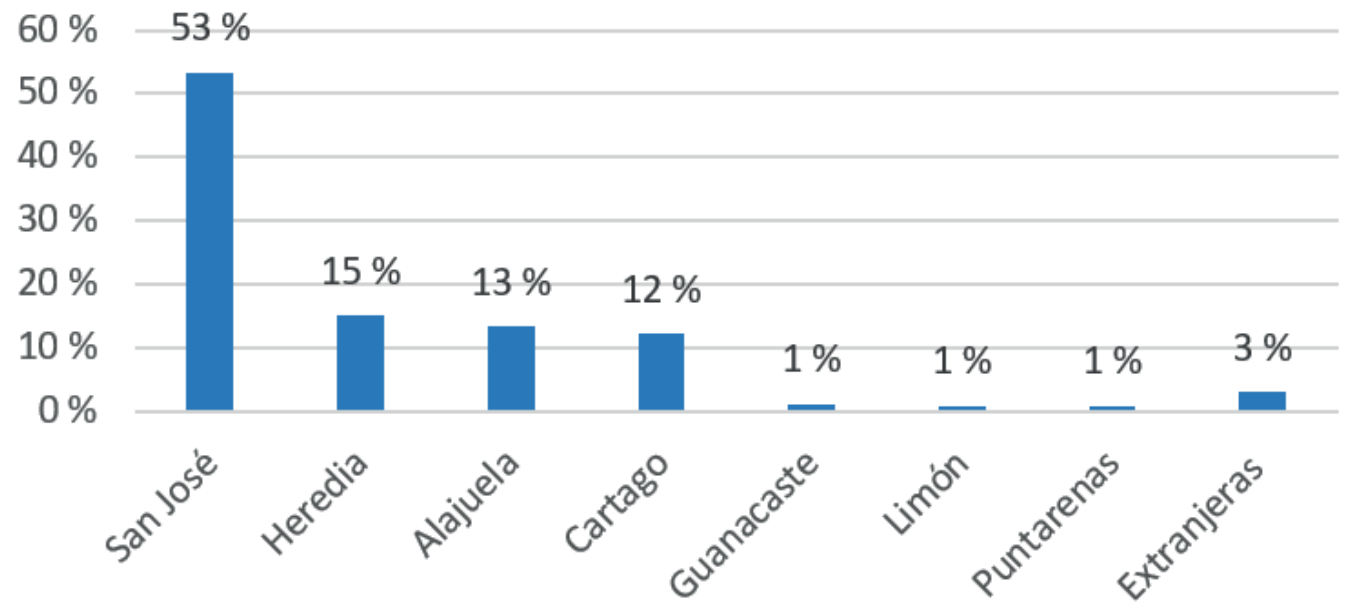

Gráfico 2. Cantones donde se ubican las empresas entrevistadas

Fuente: elaboración propia. 
Los empresarios utilizan diversos medios para hacer negocios. El 90,5\% afirmó utilizar el correo electrónico para establecer negocios, seguido de las llamadas telefónicas, encuentros personales y, en menor medida, redes sociales. El gráfico 3 muestra los medios utilizados por los empresarios para hacer negocios.

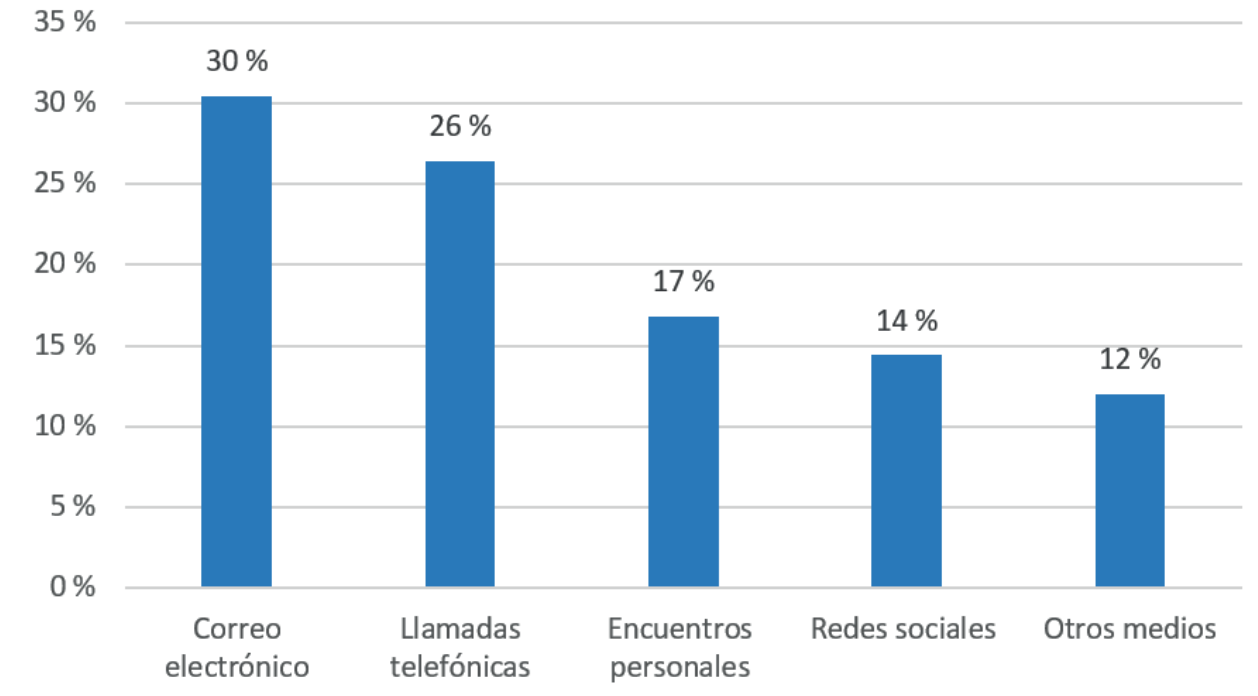

Gráfico 3. Medios utilizados para establecer negocios

Fuente: elaboración propia.

La mayoría de los empresarios poseen posee medios y habilidades tecnológicas, al menos a un nivel básico. Se observa cómo los empresarios utilizan medios electrónicos para hacer negocios; por ejemplo, correo electrónico y redes sociales. Esto es posible debido a que la totalidad de los entrevistados afirma tener facilidad y acceso a una conexión estable a Internet, así como contar con al menos una computadora.

Los encuestados indican que una plataforma de mercado virtual debería estar disponible en varios idiomas y ofrecer la opción de traducción simultánea de los mensajes, de tal forma que se facilite el proceso de concretar negocios. Ante la consulta sobre si el sitio web debe o no disponer de otros lenguajes, el 69,1\% manifestó que debe haber más opciones, mientras que $7,1 \%$ dijo no necesitar otro idioma, y a un $23,8 \%$ le parece irrelevante este tema. Un $45,2 \%$ mencionó que la información debería estar en español e inglés y, en menor medida, mencionaron el portugués, mandarín y francés.

Además, los participantes estiman que un sitio web debería tener múltiples funciones que faciliten el intercambio de bienes y servicios. Entre esas funciones señalan: establecer contacto 
con socios comerciales (35\%), aprovechar el espacio para exponer sus productos y ofertas (31\%) y realizar la valoración del mercado y competencia $(24 \%)$. Asimismo, el sitio debería permitir realizar consultas y recibir y enviar cotizaciones. Destaca también la importancia de poder realizar compras y negociaciones con el respaldo de la plataforma. El gráfico 4 muestras las funciones que debería tener el sitio web.

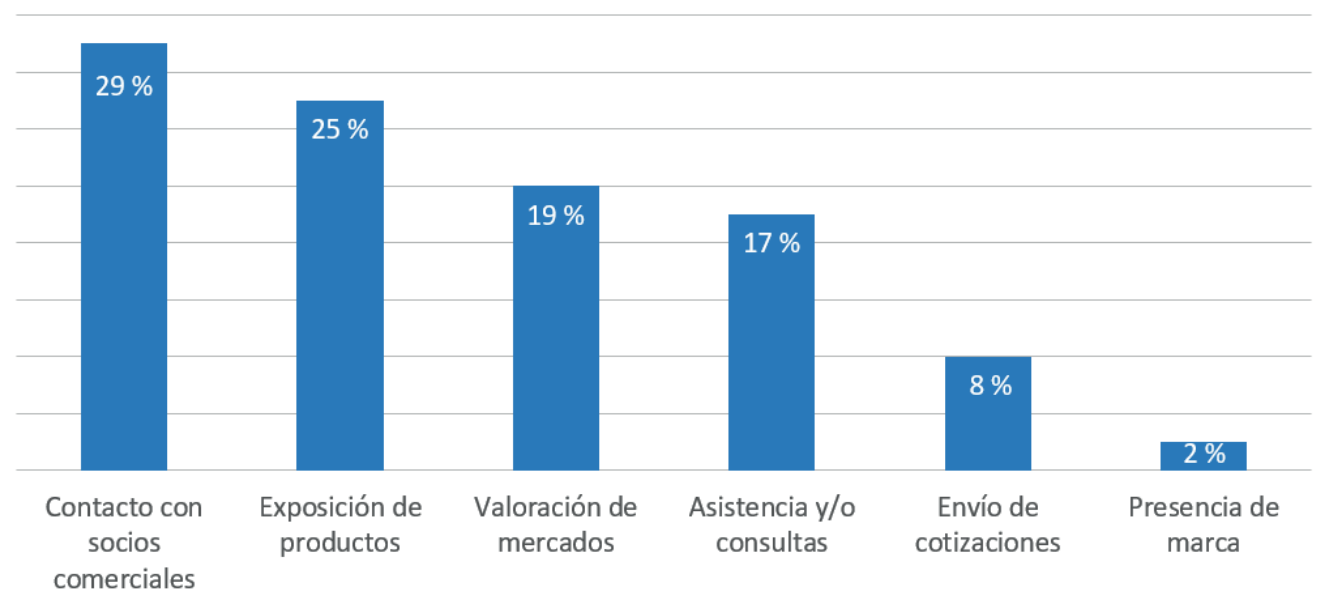

Gráfico 4. Funciones que debería tener un mercado virtual Fuente: elaboración propia.

A pesar de ser empresarios con habilidades tecnológicas y que han utilizado los mercados virtuales, es muy poca la cantidad de negocios que cierran por medio del mercado virtual. Un 95,2 \% de las personas entrevistadas aún no han concretado ningún negocio y solo el 4,8 \% restante sí lo ha logrado. Las razones por las cuales no han concretado negocios son: no encontró contraparte para negociar (48,8 \%); encontró alguna empresa pero no recibió respuestas satisfactorias a las consultas realizadas (12,2\%); estableció contacto con la otra empresa pero no fue constante en el seguimiento de la oportunidad de negocio $(2,4 \%)$. Asimismo, un 36,6 \% atribuyó el no establecimiento de negocios a otros motivos, principalmente asociados a no lograr acuerdos en las condiciones o precio de venta, no tener capacidad instalada para dar soporte al pedido realizado, no tener capital de trabajo suficiente para ofrecer el pedido, solo poder vender al contado y no ofrecer crédito. El gráfico 5 presenta las razones por las cuales no se han concretado negocios por medio de un mercado virtual. 


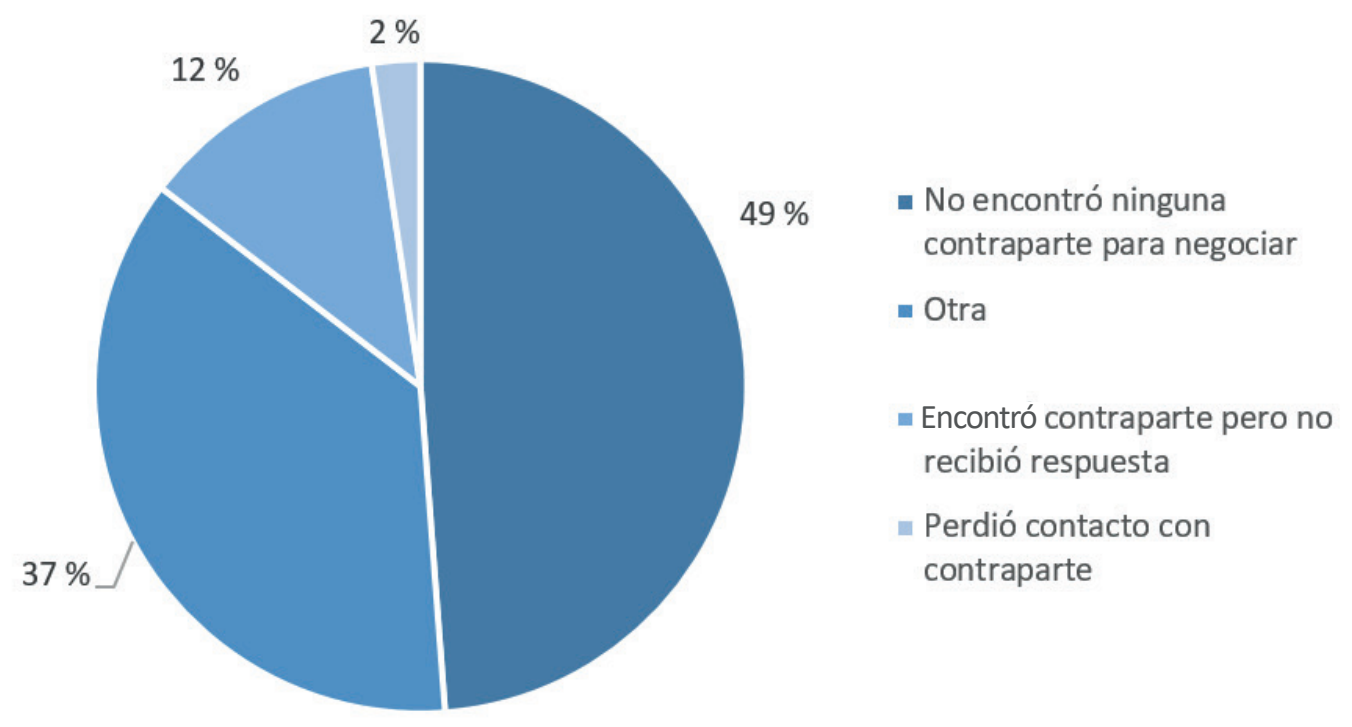

Gráfico 5. Razones que no han permitido establecer negocios por medio de mercados virtuales Fuente: elaboración propia con base en los resultados de la encuesta aplicada.

\section{b) Análisis comparativo de las plataformas de mercado virtual}

Para el análisis comparativo de las plataformas, se tomaron en cuenta ocho mercados virtuales y varios directorios empresariales, principalmente a nivel internacional. El cuadro 1 muestra cada una de las herramientas analizadas.

En primera instancia, se debe destacar la diversidad de directorios y guías comerciales disponibles, algunas gratuitas o con costos accesibles, generadas por iniciativas públicas o privadas, especializadas en uno o varios sectores, tales como industria, agricultura, automotriz, entre otros. Por lo general, estos se presentan en sitios nada amigables con el usuario y no permiten agregar o buscar información de forma ágil, únicamente los desarrolladores tienen la capacidad de realizar esta acción y lo hacen mediante una nueva publicación. La actualización de la información también es un elemento a considerar, dado que, por lo general, los datos de contacto no se actualizan de forma regular. A continuación, se describe cada una de las plataformas analizadas en la investigación:

En Costa Rica, desde el año 2000, Procomer desarrolló la plataforma electrónica Marketplace, con el propósito de generar encadenamientos y apoyar a las pymes (Procomer, 2015). Los principales beneficios de Marketplace son la reducción de costos, la expansión del alcance de la empresa y la disminución del tiempo en negociaciones. Además, esta plataforma dinamiza los procesos empresariales gracias a la implementación de las tecnologías disponibles 
Revista de Política Económica y Desarrollo Sostenible • EISSN: 2215-4167

Vol. 4 (2) • Enero-Junio, 2019: 1-19

Fonseca Hernández

Porras Quesada

DOI: http://dx.doi.org/10.15359/peds.4-2.1

Vargas Chavarría

URL: http://www.revistas.una.ac.cr/politicaeconomica

Villalobos Solano

para facilitar el contacto con empresarios, establecimiento de negocios, solicitud de cotizaciones u órdenes comerciales, entre otros aspectos (Dobles, 2015).

En cuanto al registro en la herramienta, la información solicitada es fácil de suministrar, tiene posibilidades de inscribirse como proveedor y comprador (en perfiles separados), realizar ventas rápidas, observar productos, suplidores, comprar a clientes potenciales, buscar productos principales (por categoría y ubicación geográfica) y ubicar las últimas 50 ventas. A nivel general, la navegación en la herramienta es sencilla, su presentación no es sobrecargada y cuenta con opciones de fácil comprensión.

Cuadro 1. Comparativo de herramientas de mercado virtual

\begin{tabular}{|c|c|c|c|c|c|c|c|c|c|}
\hline Herramientas & País & Idioma & Costo & $\begin{array}{c}\text { Pri/ Púb } \\
\left({ }^{*}\right) \\
\end{array}$ & Diseño & $\begin{array}{l}\text { Diversifica- } \\
\text { ción }\end{array}$ & Compras & $\begin{array}{l}\text { Publi- } \\
\text { cidad }\end{array}$ & Dirección electrónica \\
\hline $\begin{array}{l}\text { Directorios } \\
\text { empresariales: } \\
\text {-Guía Industrial, } \\
\text {-Directorio de Expor- } \\
\text { tadores } \\
\text {-Importadores de } \\
\text { Costa Rica }\end{array}$ & $\begin{array}{l}\text { Costa } \\
\text { Rica }\end{array}$ & $\begin{array}{c}\text { Espa- } \\
\text { ñol }\end{array}$ & Gratis & Ambos & $\begin{array}{l}\text { Poco } \\
\text { amigable }\end{array}$ & $\begin{array}{c}\text { Especia- } \\
\text { lizado en } \\
\text { sectores }\end{array}$ & No & No & $\begin{array}{l}\text { De acuerdo con cada } \\
\text { herramienta mencio- } \\
\text { nada }\end{array}$ \\
\hline $\begin{array}{l}\text { Marketplace de } \\
\text { Procomer }\end{array}$ & $\begin{array}{l}\text { Costa } \\
\text { Rica }\end{array}$ & Inglés & Gratis & Público & $\begin{array}{l}\text { Poco } \\
\text { amigable }\end{array}$ & No & No & Sí & $\begin{array}{l}\text { www.servicios.proco- } \\
\text { mer.go.cr/marketplace/ } \\
\text { index.php }\end{array}$ \\
\hline ConnectAmericas & Regional & $\begin{array}{c}3 \text { idio- } \\
\text { mas }\end{array}$ & Gratis & Privado & Amigable & Sí & No & Sí & $\begin{array}{l}\text { www.connectamericas. } \\
\text { com }\end{array}$ \\
\hline eMarketServices & $\begin{array}{l}\text { Unión } \\
\text { Europea }\end{array}$ & Inglés & Gratis & Público & $\begin{array}{l}\text { Poco } \\
\text { amigable }\end{array}$ & Sí & No & No & $\begin{array}{l}\text { www.emarketservi- } \\
\text { ces.es }\end{array}$ \\
\hline Allactiontrade & Japón & $\begin{array}{c}11 \\
\text { idio- } \\
\text { mas }\end{array}$ & Gratis & Privado & Amigable & Sí & Sí & Sí & $\begin{array}{l}\text { www.allactiontrade. } \\
\text { com }\end{array}$ \\
\hline AsianProducts & Taiwán & Inglés & Gratis & Privado & Amigable & $\begin{array}{l}\text { Diversi- } \\
\text { dad de } \\
\text { productos, } \\
\text { especializa- } \\
\text { do en Asia }\end{array}$ & Sí & Sí & $\begin{array}{l}\text { www.asianproducts. } \\
\text { com }\end{array}$ \\
\hline Ec Plaza & $\begin{array}{l}\text { Corea } \\
\text { del Sur }\end{array}$ & Inglés & Gratis & Privado & Amigable & Sí & Sí & Sí & www.ecplaza.net \\
\hline EC21 & $\begin{array}{l}\text { Corea } \\
\text { del Sur }\end{array}$ & $\begin{array}{l}\text { Inglés, } \\
\text { corea- } \\
\text { no }\end{array}$ & Gratis & Privado & $\begin{array}{l}\text { Poco } \\
\text { amigable }\end{array}$ & Sí & No & Sí & www.ec21.com \\
\hline B2B de Pro México & México & $\begin{array}{c}\text { Espa- } \\
\text { ñol }\end{array}$ & Gratis & Público & Amigable & Sí & No & No & $\begin{array}{l}\text { www.hechoenmexico- } \\
\text { b2b.com }\end{array}$ \\
\hline
\end{tabular}

Fuente: elaboración propia con base en la investigación realizada.

Nota $\left(^{*}\right)$ : Pri: privada; Púb: pública 
En el caso de América Latina y el Caribe, el Banco Interamericano para el Desarrollo (BID), con el apoyo de Google, DHL, Visa y Alibaba, desarrolló ConnectAmericas, la cual se describe como "una red social empresarial de las Américas dedicada a promover el comercio exterior y la inversión internacional" (BID, 2016, p. 1). La plataforma pretende fortalecer los negocios de las pymes mediante el enlace con clientes, proveedores e inversionistas y brinda información sobre procedimientos y regulaciones del comercio internacional, así como opciones de financiamiento disponibles.

ConnectAmericas se encuentra disponible en tres idiomas: inglés, español y portugués y su registro es gratuito. Al momento del registro se realiza una revisión de la información indicada en el perfil y se autoriza o no la inscripción; además, en ciertos casos otorga un símbolo de autentificación junto al nombre de cada empresa, lo que da mayor confiabilidad a los usuarios. En esta plataforma no es posible realizar compras, pero las opciones de búsqueda son muy específicas, lo que facilita la realización de esta tarea.

El eMarketservices es una herramienta que motiva a las empresas a proyectarse a nivel internacional; es impulsado por el Instituto Español de Comercio Exterior (ICEX), un organismo público, y se encarga de promover la internacionalización de las empresas, especialmente por medio de la herramienta. La plataforma recoge la información de mercados electrónicos, empresas, instituciones gubernamentales y particulares, pero no permite realizar compras o ventas de bienes (ICEX, 2015).

En cuanto a la herramienta Allactiontrade, esta pretende establecer una plataforma para crear oportunidades de negocios y promover en línea sus bienes y servicios. Cuenta con más de 60 categorías y 100 subcategorías de productos (Allactiontrade, 2015). La navegación en el sitio es sencilla, posee gran variedad de productos y categorías que permiten delimitar la búsqueda y encontrar específicamente lo que busca cada usuario. Esta herramienta cuenta con la opción de traducir la página.

Por su parte, AsianProducts es un sitio web disponible únicamente en inglés, con gran variedad de productos manufacturados en todo el mundo. Su navegación es amigable, al permitir la visualización de las categorías, productos, usuarios y otros aspectos de forma sencilla, haciendo la información de fácil acceso (Asianproducts, 2015).

El mercado virtual Ecplaza, tiene una presentación ordenada que facilita la visualización, gracias a la gran cantidad de imágenes de los productos disponibles y los banners con movimiento. Ofrece el servicio de compra y venta de bienes, lo que resulta de interés para los usuarios. Posee una función útil e innovadora, siendo posible el envío de muestras de los productos a los compradores (EcPlaza Network Inc., 2015).

La plataforma EC21 cuenta con más de un millón de empresas inscritas, tres millones de productos y millones de visitas mensuales (KITA, 2015). Sin embargo, es posible clasificar la página como poco amigable, debido a que su sitio web principal cuenta con gran cantidad de información, por lo que en ocasiones los datos no cargan con velocidad. 
B2B de ProMéxico fue creado para promover los bienes y servicios mexicanos a nivel internacional y atraer mayor inversión extranjera. Entre los beneficios que ofrece B2B están: amplia promoción de las empresas, contacto directo con empresarios internacionales, fácil acceso a la herramienta desde cualquier país, menores costos (no pago de comisiones), ampliación de cartera de clientes (ProMéxico, 2015). Para poder realizar el registro de las empresas en dicha plataforma es necesario cumplir con requisitos previos, una vez completada la información, será sometido a una evaluación de ProMéxico para la su verificación, aspecto diferenciador que da confianza al usuario, pues en las otras plataformas la inscripción carece de mayores controles o medios de verificación de la información suministrada, que comprueben la existencia real de la empresa.

Esta es una plataforma amigable, de fácil visualización, cuenta con un índice de productos en su página inicial y rápido acceso a los productos nuevos. Este aspecto es de gran importancia para los exportadores, ya que se puede obtener información por tipo de bien, por región y así como por las oportunidades comerciales que tengan a nivel nacional e internacional.

Las plataformas analizadas buscan bajar los costos de intermediación y disminuir barreras al comercio al acercar los compradores a los vendedores. Mayoritariamente son plataformas creadas por usuarios privados y ofrecen una gran variedad de productos y servicios. A nivel general, las plataformas ofrecen la información en varios idiomas, siendo el inglés el que predomina. Ofrecen sus servicios de forma gratuita. Solo cuatro plataformas ofrecen la posibilidad de realizar compras, las demás brindan únicamente el contacto, facilitando la interacción entre la oferta y la demanda.

\section{Discusión}

Producto de la información recabada mediante la encuesta aplicada y el análisis de las funcionalidades que tienen los diferentes mercados virtuales consultados, a continuación, se analizan los factores competitivos y elementos funcionales que deberían incorporar o mejorar las plataformas de mercados virtuales para hacerlas más accesibles y adaptadas a las necesidades de las pymes. En primera instancia se propone establecer una estrategia de corto y mediano plazo general, considerar elementos de diseño e idiomas de la plataforma, así como aspectos a incluir en el registro y perfil de las empresas, entre otras funcionalidades. Cabe mencionar que dichos elementos fueron validados por expertos de la Universidad Nacional.

En primera instancia, es imperativo diseñar una estrategia de mejora de la herramienta como tal, a corto y mediano plazo. Esta debería indicar claramente cuáles son las necesidades primordiales por atender, los cambios por ejecutar y los mecanismos para la retroalimentación por parte de los usuarios. Esta estrategia podría desarrollarse por etapas y contemplar los elementos que a continuación se describen.

En lo que respecta al diseño e idioma de la herramienta, debe contemplar un atractivo visual y facilidad de navegación. La plataforma debería estar disponible en varios idiomas, 
al menos tres: español, inglés y portugués. Pudiéndose habilitar, en una etapa posterior, la traducción al mandarín y al francés.

En relación con el proceso de registro de los usuarios, este debe ser simple, solicitar información básica pero obligatoria y contar con un mecanismo de validación por parte del administrador de la plataforma, para constatar al menos que la empresa que se registre es real; dicha información debería actualizarse de forma obligatoria cada cuatro o seis meses. Además, una vez se ha completado el registro, se recomienda realizar el envío de un manual con los principales elementos y beneficios de la plataforma, de modo que se facilite la navegación. Es necesario permitir que los usuarios puedan registrarse como compradores y vendedores, así como habilitar la opción de ofrecer múltiples productos o servicios según su giro de negocios.

Como paso posterior al registro de usuario básico, se recomienda solicitar un segundo registro más detallado para crear un perfil tipo catálogo, en el cual las diferentes empresas puedan contar, de forma gratuita, con un perfil básico, que incluya información sobre sus productos o servicios y demás detalles que deseen compartir con quienes estén interesados en comprarlos o venderlos. Podrían incluirse aspectos básicos como logos, fotografías o descripciones de productos. Se recomienda también asignar una sección para que los usuarios indiquen las certificaciones nacionales e internacionales que poseen y expliquen en qué consiste cada una.

El mercado virtual deberá tener la capacidad para recibir oferentes y demandantes de diferentes industrias y sectores. De tal forma que sea posible generar encadenamientos comerciales de cualquier índole. En este sentido, debe habilitarse la mayor cantidad de categorías de negocios posible e, incluso, generar opciones para crear una nueva área, si así se requiere.

La nacionalidad de las empresas inscritas debería estar abierta a cualquier país, no debe limitarse la inscripción únicamente a negocios de Costa Rica. La plataforma debe estar habilitada para recibir afiliaciones de empresas de otras naciones que puedan comprar y ofertar productos diversos en suelo nacional.

A continuación, se detallan las funcionalidades principales que debe incorporar la plataforma de mercado virtual, las que se detallan a continuación:

Tener un espacio para realizar negociaciones y compras en un ambiente seguro dentro de la plataforma. Uno de los elementos interesantes que ofrecen las plataformas analizadas es que permiten la posibilidad de realizar negociaciones comerciales y pagos en línea, siendo la plataforma un intermediario; para lo que se utiliza pago con tarjeta de crédito, así como PayPal. Deberían, de igual forma, considerarse opciones de compras e intercambio tipo trueque ${ }^{5}$, envío de mercadería e incluso envío de muestras sin valor comercial para iniciar la relación con entre los compradores. El sistema debe ofrecer facilidad, rapidez, seguridad y respaldo para realizar una orden de compra en línea.

Establecer un sistema de búsqueda avanzado, para que las empresas puedan filtrar y ser

5 Intercambio de productos y servicios con valores similares sin que medie pago en efectivo por estos. 
ubicadas con facilidad de acuerdo al tipo de productos o servicios que ofertan y requieren, así como por bienes complementarios o ubicación geográfica, entre otros filtros que podrían aplicarse para mejorar el acceso a la información.

Establecer un sistema de notificaciones al recibir mensajes o solicitudes de reunión o cotización de contrapartes. Puede integrarse el envío de un correo electrónico a la cuenta con la que se creó el perfil de registro, así como una alerta al momento de ingresar a la página. Es posible integrarlo con algún otro medio de comunicación por redes sociales.

Asimismo, se sugiere agregar la opción de asistencia en línea, lo cual genera una respuesta en tiempo real, para potenciar la confianza de los usuarios y el adecuado uso de la herramienta, en caso de que requieran asistencia remota debido a dificultades técnicas. Para el adecuado manejo de la plataforma, debe contarse con un equipo de trabajo técnico a nivel de comercio exterior e informático, para resolver las consultas o problemas que se generen.

También se debería complementar la herramienta con una aplicación para dispositivos móviles, que permita a los usuarios ingresar en cualquier momento con facilidad; así como atender solicitudes y generar consultas de forma inmediata. En principio la aplicación debería permitir realizar los mismos procesos que la página web.

Se considera vital desarrollar un mecanismo para obtener información y estadísticas de uso en relación con la cantidad de negocios generados a partir de la herramienta, así como el aprovechamiento de las principales funciones del mercado virtual utilizadas por los usuarios. Además, debe perseguirse un objetivo de mejora continua, para habilitar la retroalimentación por parte de los usuarios, en cuanto a conocer su opinión y requerimientos.

Respecto a los costos y el financiamiento de la plataforma, esta debería ser de inscripción gratuita y ser financiada por instituciones públicas, o bien, por empresas que busquen mayor posicionamiento de sus productos. Las herramientas analizadas no cobran por la inscripción a las plataformas, lo cual es una estrategia correcta, debido al mercado pyme al que se dirige y también es atractivo para ampliar la afiliación constante de nuevos usuarios. Para su mantenimiento, se recomienda generar ingresos por medio de publicidad, tanto de organizaciones que prestan servicio para las pymes, tipo bancos o asesorías, como de los mismos usuarios inscritos en la plataforma, quienes buscan una mayor visualización de sus productos, promociones o estrategias de comunicación.

Se debe diseñar una estrategia de divulgación y promoción del mercado virtual, la cual incluya el diseño de logos, desplegables y otros materiales publicitarios que difundan la información principal de la herramienta. Se recomienda la búsqueda de aliados institucionales tanto a nivel nacional como internacional y firmar alianzas estratégicas con bancos, aseguradoras $\mathrm{y}$ otros entes que ofrecen servicios para pymes.

Adicionalmente, debe contemplarse la realización de capacitaciones y talleres sobre los mercados virtuales, sus beneficios y oportunidades, complementado temas generales del 
mercado internacional y el e-commerce, con el fin de impulsar el uso de la herramienta en pro de maximizar oportunidades comerciales de los usuarios. Las capacitaciones pueden ofrecerse de forma presencial, bimodal o virtual.

Adicionalmente, se recomienda realizar con cierta periodicidad una depuración y actualización de la base de datos, para facilitar los negocios, la generación de estudios futuros y el mantenimiento de la confianza por parte del usuario. Por último, es de vital importancia que la plataforma tenga respaldos de la información en servidores seguros.

\section{Conclusiones}

Los mercados virtuales son una línea de acción que permite apoyar el área estratégica el desarrollo de plataformas tecnológicas de comunicación, vigente en la política de Fomento al Emprendimiento en Costa Rica 2014-2018. Dichas plataformas ahorran tiempo, costos importantes y amplían el mercado nacional e internacional al que pueden acceder las empresas costarricenses. En este sentido, se recomienda como acción dentro de política de apoyo al sector pyme y emprendimiento, el mejoramiento de la plataforma Marketplace de Procomer, o bien, el desarrollo de una nueva plataforma que encuentre las áreas operativas del Ministerio de Economía, Industria y Comercio con la Promotora de Comercio Exterior.

La plataforma a reforzarse o crearse debería incorporar o mejorar los siguientes elementos competitivos y funcionales para hacerla más accesibles y adaptada a las necesidades de las pymes: diseñar una estrategia de mejora a corto plazo; renovar el diseño y ofrecer opciones de idioma; mejorar el registro y perfil de las empresas a registrar; habilitar la plataforma a diferentes industrias, sectores y nacionalidades; incluir funcionalidades tales como poder realizar negociaciones y compras con el respaldo de la plataforma, contar con un sistema de notificaciones, asistencia en línea, información y estadísticas de uso, así como estar disponible en diversos dispositivos; el costo debería ser gratuito y financiar los gastos operativos por medio de publicidad. También es necesario establecer una estrategia de divulgación y promoción del mercado virtual, realizar una depuración y actualización constante de la base de datos y contemplar la generación de respaldos frecuentes de la información.

\section{Referencias bibliográficas}

España Exportaciones e Importaciones [ICEX]. (2015). eMarket services. Recuperado de http://www.emarketservices.es/icex/cda/controller/ pageemarket/0,3200,1480591 $14870301518180 \quad 0,00$. html

Allactiontrade. (2015). Allactiontrade. Recuperado de www.allactiontrade.com/

Asianproducts. (2015). Asianproducts Service. Recuperado de https://www.asianproducts.com/ UserGuide 
Banco Interamericano de Desarrollo [BID]. (2016). Banco Interamericano de Desarrollo. Recuperado de https:/connectamericas.com/es/content/sobre-connectamericas

Bonilla, L., y Mesén, L. B. (2013). Condiciones actuales del financiamiento de las pymes costarricenses. Tec Empresarial, 7(2), 29-39. Recuperado de https://dialnet.unirioja.es/ descarga/articulo/4456948.pdf

Castro, O. (2014). Elementos culturales en los procesos de negociación internacional. Caso China. Suma de Negocios, 5(12), 143-147. Recuperado de https://ac.els-cdn.com/

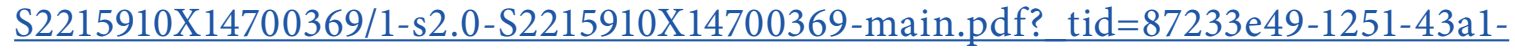
8ac0-050c1726a0d5\&acdnat=1536254694 0ad0de5e8fb95477627a2d41b7858b51

Chaves, M. y Fonseca, R. (2015). Emprendedurismo en Costa Rica: estancamiento en la transición a la innovación. Economía y Sociedad, 20(48), 1-21. Recuperado de http://www. revistas.una.ac.cr/index.php/economia/article/view/7550/7931

Dobles, R. (2015). La herramienta del Marketplace: Aspectos generales e históricos. Recuperado de https://idx.com.co/e-commerce-o-marketplace-500b7191aa06

EcPlaza Network Inc. (2015). EcPlaza Network Inc. Recuperado de https://www.ecplaza.net/ $\underline{\text { aboutus }}$

Espinoza, F. y Flores, J. (2018). Estrategias de comercio electrónico: oportunidad de negocios en las empresas del sur de Sonora. Revista Iberoamericana de las Ciencias Computacionales e Información, 7(13), 1-16. Recuperado de http://www.reci.org.mx/index.php/reci/article/ view/78/367

Figal, L. y Maffioli, A. (2016). Evaluación de impacto de políticas de innovación en América Latina y el Caribe: hacia una nueva frontera. En La política de innovación en América Latina y el Caribe nuevos caminos (pp. 237-278). Washington, Estados Unidos: Banco Interamericano de Desarrollo. Recuperado de https://webimages.iadb.org/publications/ spanish/document/La-pol\%C3\%ADtica-de-innovaci\%C3\%B3n-en-Am\%C3\%A9ricaLatina-y-el-Caribe-Nuevos-caminos.pdf

Fonseca, R., y Chaves, M. (2015). Política Pública "Costa Rica Emprende”: Uma Análise dos resultados. Revista de Relaciones Internacionales, (87.2), 93-110. Recuperado de http:// www.revistas.una.ac.cr/index.php/ri/article/view/6693/6829

Hernández, R. (2017). Impacto de las capacidades de interneten la gestión, la operativa internacional y la internacionalización de las PYMES exportadoras. Ocho estudios de caso en Costa Rica (Tesis de doctorado inédita), Universidad de Sevilla, España. Recuperado de https:// idus.us.es/xmlui/bitstream/handle/11441/61116/Tesis\%20Rosmery\%20versi\%C3\%B3n\%20 $\underline{\text { final } \% 2010 \% 20 \mathrm{de} \% 20 \text { enero.pdf?sequence }=1 \& \text { isAllowed }=\mathrm{y}}$ 
Hurtado, V. (2009). Diseño y construcción de Electronic Marketplace (Tesis de grado inédita). Universidad de Chile, Chile:. Recuperado de http://www.tesis.uchile.cl/tesis/uchile/2009/ cf-hurtado gv/pdfAmont/cf-hurtado gv.pdf

KITA. (2015). Korea International Trade Association. Recuperado de http://www.ec21.com/ html/ec/AU/AU Company Overview.html

Lavios, J., Olmo, R., Mariscal, M. y García, S. (2005). Mercados virtuales. Situación actual y retos para el futuro. España: Asociación para el Desarrollo de la Ingeniería de Organización.

Martínez, K. y Vásquez, I. (2018). El rol de la gerencia en la adopción del comercio electrónico: El caso de las mipymes costarricenses. TEC Empresarial, 12(2), 45-53. Recuperado de http://181.193.125.13/index.php/tec empresarial/article/view/3720/3341

MEIC. (2015). Estado de Situación de las PYMES en Costa Rica. San José, Costa Rica: Ministerio de Economía, Industria y Comercio. Recuperado de http://reventazon.meic. go.cr/informacion/estudios/2016/PYMES/informe.pdf

MEIC. (2017). Estado de Situación de las PYMES en Costa Rica. Costa Rica: Ministerio de Economía, Industria y Comercio. Recuperado de: http://reventazon.meic.go.cr/ informacion/pyme/2017/informe.pdf

Medina-Chicaiza, P., Tibanta-Narváez, E. y Pazmay-Pazmay, P. (2017). Aproximación a una propuesta metodológica para la construcción de centros virtuales de comercialización para las PYMEs. Revista Espacios, 38 (53), 1-8. Recuperado de http://www.revistaespacios.com/ a17v38n53/a17v38n53p01.pdf

Miño, G., Napoleón, A. y Orozco, J. (2017). Tecnologías de información y comunicación como herramienta para incrementar el posicionamiento empresarial caso Inpapel. Revista mktDescubre, 9, 95-105. Recuperado de http://revistas.espoch.edu.ec/index.php/ mktdescubre/article/view/38/32

Nieto, P. (2016). El comercio electrónico y la contratación electrónica: Bases del mercado virtual. Revista Foro Jurídico, 15, 54-76. Recuperado de http://revistas.pucp.edu.pe.una.idm.oclc. org/index.php/forojuridico/article/view/19835/19879

Petronio, M. (2003). Mercados Virtuales (Tesis de grado inédita). Instituto Tecnológico: Argentina.

Promotora de Comercio Exterior [Procomer]. (2015). Análisis sobre la evolución de las exportaciones de Costa Rica. Recuperado de http://www.comex.go.cr/media/1990/analisissobre-la-evolucion-de-las-exportaciones-de-cr-24jul2015.pdf

Promotora de Comercio Exterior [Procomer]. (2015). Marketplace. Recuperado de http:// servicios.procomer.go.cr/marketplace/login.php 
Programa Estado de la Nación. (2018). Informe Estado de la Nación 2018. San José, Costa Rica: PEN-CONARE. Recuperado de https://www.estadonacion.or.cr/2018/assets/en2018.pdf

ProMéxico. (2015). ProMéxico Demo exportadores. Recuperado de http://www.promexico.gob. $\mathrm{mx} / \mathrm{es} / \mathrm{mx} /$ home

Reyes, C. y Zamora, J. (2009). Las TICs y la Asociatividad Comercial: el Caso de la Ruta del Vino del Maule. Chile: Universidad de la Frontera. Recuperado de https://www.researchgate.net/ publication/221419376 Las TICs y la Asociatividad Comercial El Caso de la Ruta del Vino del Maule

Romo, D. y Andel, G. (2005). Sobre el concepto de competitividad. Revista Comercio Exterior, 55(3), 200-214. Recuperado de http://revistas.bancomext.gob.mx/rce/magazines/76/1/RCE.pdf

Ruano, E. (2016). Red regional de emprendimiento del Cauca: avances y desafíos de un instrumento de política pública. Revista Ciencias Estratégicas, 24(35), 181-197. Recuperado de https://revistas.upb.edu.co/index.php/cienciasestrategicas/article/download/7603/6935

Salas, M., Martínez, R. y Chamba, L. (2018). Evolución de las pymes. Observatorio de la Economía Latinoamericana, (abril), 1-19. Recuperado de https://www.eumed.net/rev/oel/2018/04/ evolucion-pymes-ecuador.html

Sarcos, H. e Indriago, F. (2016). Libertad de maniobras de procesos de negociaciones en américa latina. CICAG, 13(2), 363-372.

Sáurez, M.yFonseca, R.(2014). Diagnóstico socioeconómicoy perspectivas deinternacionalización: Estudio de caso 14 microempresarios de la Región Brunca. Revista en Relaciones Internacionales, 87(2), 59-79. Recuperado de http://www.revistas.una.ac.cr/index.php/ri/ article/view/6687/6823

Tabares, S., Anzo, E. y Vanegas, J. (2016). Internationalization and SMEs: An analysis of manager characteristics. Revista Espacios, 37 (27), 21-42. Recuperado de http://www.revistaespacios. com/a16v37n27/16372722.html

Universidad de Costa Rica[UCR]. (2018). Informe de resultados III Encuesta Nacional de la Micro, pequeña y mediana empresa en Costa Rica 2018. Costa Rica: Observatorio del Desarrollo, Universidad de Costa Rica. Recuperado de http://reventazon.meic.go.cr/informacion/ pyme/2018/estadopyme/informe.pdf

Varado, I., Cortez, V. y Sánchez, F. (2018). Universalizar las pymes: un proyecto importante en materia de comercio internacional. RECIMUNDO, 2(1), 971-985. Recuperado de http:// recimundo.com/index.php/es/article/view/212/pdf

Varian, H. (1998). Análisis Microeconómico. España: Antoni Bosch. 